\title{
Analysis of the Effects of Rainfall Intensity into the Performance of Video Conferencing
}

\author{
Charles Duhujamahoro, Celestin Twizere, Patrick Mazimpaka, Thierry Ony Shema, \\ Nkurunziza Pascal \\ Electrical and Electronics Engineering Department, University of Rwanda, Butare, Rwanda \\ Email: cduhujamahoro@ur.ac.rw, celestintwizere@gmail.com,chemeerry3@gmail.com,paka05@yahoo.fr, \\ nkurpas88@gmail.com
}

Received 21 October 2015; accepted 1 February 2016; published 5 February 2016

Copyright (C) 2016 by authors and Scientific Research Publishing Inc.

This work is licensed under the Creative Commons Attribution International License (CC BY).

http://creativecommons.org/licenses/by/4.0/

(c) (i) Open Access

\begin{abstract}
Attenuation due to rain is an important limitation in the designing of the microwave communication links operating at frequencies above $10 \mathrm{GHz}$. Video conferencing performs over high-speed link via high-speed satellite (WINDS satellite) transmission in the Ka-band which is vulnerable to attenuation due to rain. Using the ITU-R proposed models for the prediction of rain attenuation gives a good estimation for the microwave propagation loss caused by rain for the temperate regions, but it underestimates the rain attenuation prediction for the monthly variation of rain rate by averaging annual rain rate for particular region. This study was carried out at the University of Rwanda/Huye Campus. The rain rates were predicted for 0.01 percentage of time from rainfall data measured during two months of July and August using the RD-80 Joss and Waldvogel Disdrometer. From the data collected and analyzed, compared with ITU-R model, we can see that where Rwanda region is situated in region $K$, what is different from the obtained results because the data are collected during months that coincide with a dry season.
\end{abstract}

\section{Keywords}

Attenuation, Rain Rate, Video Conferencing, ITU-R Model

\section{Introduction}

Video conferencing is a communication technology that requires transmission of video and audio data to connect users anywhere in the world as if they were in the same room [1]. This technology is highly growing worldwide in different fields but most popular in business, academics and medicine fields since it allows meetings, distance learning or telemedicine to be held without the need for all the participants to travel to a single location, so it saves time and money [1]. Almost any organization that holds meetings for people from different

How to cite this paper: Duhujamahoro, C., Twizere, C., Mazimpaka, P., Shema, T.O. and Pascal, N. (2016) Analysis of the Effects of Rainfall Intensity into the Performance of Video Conferencing. World Journal of Engineering and Technology, 4, 71-81. http://dx.doi.org/10.4236/wjet.2016.41008 
locations, whether across town or around the world, can make use of video conferencing. Video conferencing also requires a connection to the communication system which in the $21^{\text {st }}$ century is usually the Internet but might also be a satellite based system, a broadcast signal or other communication technologies [2]. Nowadays, due to the congestion of the radiofrequency spectrum and in order to offer broader transmission channels for multimedia applications, satellite communication systems are moving to higher frequency bands. Increasing number of new services including video conferencing are being promoted to Ka-band (30 GHz uplink and 20 $\mathrm{GHz}$ downlink) satellite communication systems involving especially very small aperture terminals (VSAT) [3].

However, the Ka-band vulnerability to signal attenuation under certain weather conditions, especially rain, is a drawback to this technology. Rain attenuates this frequency band by scattering and absorption of electromagnetic waves by drops of liquid water. This is more severe in the tropical region where heavy convective rain dominates [4]. In case of Huye campus located at a longitude and latitude of $29.7^{\circ}$ and $2.6^{\circ}$ respectively, rain intensity varies accordingly and this may affect the transmission. Some prediction models have been proposed by ITU-R based on different regions. In this paper we compare rain attenuation predicted by models and data collected at UR-Huye campus over frequency bands allocated to video conferencing (30 GHz uplink and $20 \mathrm{GHz}$ downlink) for a two-month period of July and August 2014.

\section{Theoritical Analysis}

Tropical climate region have been defined as those having uniform average annual temperature. In addition of having uniform temperature throughout the year, tropical climate region does not experience winter at all which the terms of winter and summer essentially have no meaning [5]. Rwandan climate is categorized as tropical temperate, climates which do not involve extremes of temperature and precipitation (rain and snow). The changes between summer and winter are generally invigorating without being frustratingly extreme [6].

The primary atmospheric region affecting Ka-band communications is the troposphere, which extends from the surface to an elevation of approximately $15 \mathrm{~km}$. virtually all precipitation occurs within the troposphere, as it contains about $99 \%$ of the water vapor in the atmosphere.

The other atmospheric region of interest is the ionosphere, which extends from about $70 \mathrm{~km}$ above the surface to $1000 \mathrm{~km}$, where scintillation occurs.

Scintillation is a perturbation in the amplitude and phase of an electromagnetic signal caused by variations in the dielectric parameters of the atmosphere.

Scintillation effects occur in the region of the ionosphere $400 \mathrm{~km}$ above the surface of the earth, though Ka-band scintillation also occurs in the troposphere.

Atmospheric absorption occurs when electromagnetic waves interact with molecules in the atmosphere caused signal attenuation. These molecules will absorb some parts of the signal and the remaining will be scattered, refracted or reflected. There are various different molecules and particles contained in the atmosphere that absorbs and transmits many electromagnetic radiations with different wavelengths. Water vapor, carbon dioxide and ozone cause most of the absorption.

The influence of clouds can also be existed on an earth-space path and must also be taken into account. Clouds are composed of either water droplets or ice crystals. Ice clouds, by feature of the low dielectric constant of ice and the small size of the essential particles, are expected not to cause considerable attenuation to radio waves in the frequency range below $50 \mathrm{GHz}$ [7].

The most important weather effect for our purposes is rain, which includes wet snow and other moist precipitation which must be taken into consideration in concern for earth space communication operating at frequency band above $3 \mathrm{GHz}$, dry snow has a minimal effect on Ka-band propagation.

The rain problem is particularly important for communication link above $10 \mathrm{GHz}$. Raindrops will be a medium that absorb and scatter radio wave signal, which resulting as reduction in the transmitted signal amplitude. This condition called rain attenuation can reduce the dependability and performance of the communications link [8].

Rain events can last for an extended period of time, but the most significant attenuation due to rain occurs during relatively short periods of very intense rainfall ( for convective rain). These intense rain fades are highly localized, so terminals in the same general region often will not experience similar rain fades simultaneously. The depth of rain fades vary significantly with carrier frequency. In general, the higher the carrier frequency is, the deeper the fades get.

The international engineering and scientific community has spent years collecting data on rain and other 
weather effects and devising statistical models that reflect the observed behavior. The depth of rain fade is generally measured as a function of availability. Availability is a long-term statistical concept; it indicates the probability at which the specified parameter (in our case, attenuation due to rain) will not exceed a stated value. For example, if the availability is $99.5 \%$ and the stated attenuation is $5 \mathrm{~dB}$, this means that in the long term we expect attenuation to exceed $5 \mathrm{~dB} 0.5 \%$ of the time. Most of the time, the attenuation will be much less than this amount [8].

Rainfall of high intensity is difficult to record and measure experimentally as well as being highly variable from year to year. However, in system design it is the highest rainfall rates which are frequently of great interest. Short integration time rainfall rate is the most essential input parameter in the prediction models for rain attenuation

Several models exist for the prediction of point rainfall rate cumulative distribution, this include the work of Crane which has considerably influenced the zonal models of the ITU-R and have been used extensively in the United States, although to a lesser extent in the other parts of the world, the limit of the model being the number of station years of measurements available and not all stations fulfilled the one minute integration time requirement. The results reported by Segal also influenced the ITU-R zonal models and provided a systematic approach for obtaining a specified number of rain zones in country such as Canada. Watson et al. later mapped rain rates exceeded for $0.1 \%$ and $0.01 \%$ of an average year based on data for 400 locations within Europe. This approach has been excellent in providing high quality estimates for rain intensity and was used to update the ITU-R rain zone in Europe. The drawback of this approach is that it required a relatively high density of short integration time point precipitation measurements or measurements from which these can be derived. The topography is also not explicitly taken into account, therefore requiring a high spatial resolution for the measurement data. This approach cannot be easily used on global data due to the low spatial resolution of point measurements on a global scale and the errors that would arise from the spatial interpolation of precipitation rates for fixed annual probability levels [9].

Holmberg and Rice also developed a model for obtaining rain rate values for use in fading calculation known as Rice-Holmberg's model. The model required certain parameters like highest monthly rainfall accumulation observed in a set of 30 years period, number of thunderstorm days expected in an average year and the average annual accumulation. The thunderstorm ratio is not always readily available from local weather agencies. The model was later modified by Dutton and Dougherty to make it depend on four parameters, two of which are used to estimate the fraction of thunderstorm rain, However, it has been acknowledged that the Rice-Holmberg's method overestimates rain rates in the high availability range $(0.01 \%)$, and underestimates in the range between $0.1 \%$ and $1 \%[10]$.

Recent analysis suggests that the rain rate distribution is better described by a model which approximates a long-normal distribution at the low rates, and a gamma distribution at high rain rate. This kind of model was developed by Moupfouma and Martins which is good for both tropical and temperate climate and $1 \%$.

To estimate $R_{0.01}$ of J. Chebil's model appears suitable, it allows the usage of long time mean annual accumulation (M) at the location of interest. The power law relationship of the model is given by $R_{0.01}=\alpha M^{\beta}$ where $\alpha$ and $\beta$ are regression coefficients.

Chebil has made a comparison between some models based on measurements values of $R_{0.01}$ and $\mathrm{M}$ in Malaysia, Indonesia, Brazil, Singapore and Vietnam. He showed that this model is the best estimate of the measured data. The regression coefficient $\alpha$ and $\beta$ are defined as $\alpha=12.2903$ and $\beta=0.2973$.

Thus, using the refined Moupfouma model and Chebil model the one minute rain rate cumulative distribution is fully determined from the long term mean annual rainfall data [9].

Rain attenuation is the significant propagation impairment at $\mathrm{Ku}$ and $\mathrm{Ka}$ band frequencies. In ITU-R rain attenuation prediction model, the rain intensity at $0.01 \%$ probability level is the rain related input to the model. The model has been derived on the basis of the log-normal distribution where both point rain intensity and path attenuation distributions conform to the log-normal distribution. The model is applicable across the frequency range 4 to $55 \mathrm{GHz}$ and percentage probability range $0.001 \%$ to $10 \%$ [11].

It has been reported that the ITU-R rain attenuation prediction model result were close to the average prediction of a set of results obtained from the application of eight different methodologies.

The following procedure provides estimates of the long-term statistics of the slant-path rain attenuation at a given location for frequencies up to $55 \mathrm{GHz}$.

The following parameters are required and are highlighted by the Figure 1 . 
$R_{0.01}$ : point rainfall rate for the location for $0.01 \%$ of an average year $(\mathrm{mm} / \mathrm{h})$

$h_{s}$ : Height above mean sea level of the earth station $(\mathrm{km})$

$\theta$. Elevation angle to the satellite (degrees)

$\varphi$ : Latitude of the earth station (degrees $\mathrm{N}$ or $\mathrm{S}$ )

$f$ : Frequency of operation (GHz)

$R_{\theta}$ : Effective radius of the Earth $(8500 \mathrm{~km})$

The step by step procedure for calculating the attenuation distribution is given below.

Step 1: Determine the rain height, hR, as given in Recommendation ITU-R P.839.

Step 2: For $\theta \geq 5^{\circ}$ compute the slant-path length, Ls, below the rain height from:

$$
L_{S}=\frac{\left(h_{R}-h_{S}\right)}{\sin \theta} \mathrm{Km}
$$

For $\theta<5^{\circ}$, the following formula is used:

$$
L_{s}=\frac{2\left(h_{R}-h_{s}\right)}{\left(\sin ^{2} \theta+\frac{2\left(h_{R}-h_{s}\right)}{R_{e}}\right)^{1 / 2}+\sin \theta} \mathrm{km}
$$

If $h_{R}-h_{s}$ is less than or equal to zero, the predicted rain attenuation for any time percentage is zero and the following steps are not required.

Step 3: Calculate the horizontal projection, $L G$, of the slant-path length from:

$$
L_{G}=L_{s} \cos q \mathrm{Km}
$$

Step 4: Obtain the rainfall rate, R0.01, exceeded for $0.01 \%$ of an average year (with an integration time of 1 $\mathrm{min}$ ). If this long-term statistic cannot be obtained from local data sources, an estimate can be obtained from the maps of rainfall rate given in Recommendation ITU-R P.837. If R0.01 is equal to zero, the predicted rain attenuation is zero for any time percentage and the following steps are not required.

Step 5: Obtain the specific attenuation, $\gamma \mathrm{R}$, using the frequency-dependent coefficients given in Recommendation ITU-R P.838 and the rainfall rate, R0.01, determined from Step 4, by using:

$$
g(R)=k\left(R_{0.01}\right)^{a} \mathrm{~dB} / \mathrm{Km}
$$

Step 6: Calculate the horizontal reduction factor, $r 0.01$, for $0.01 \%$ of the time:

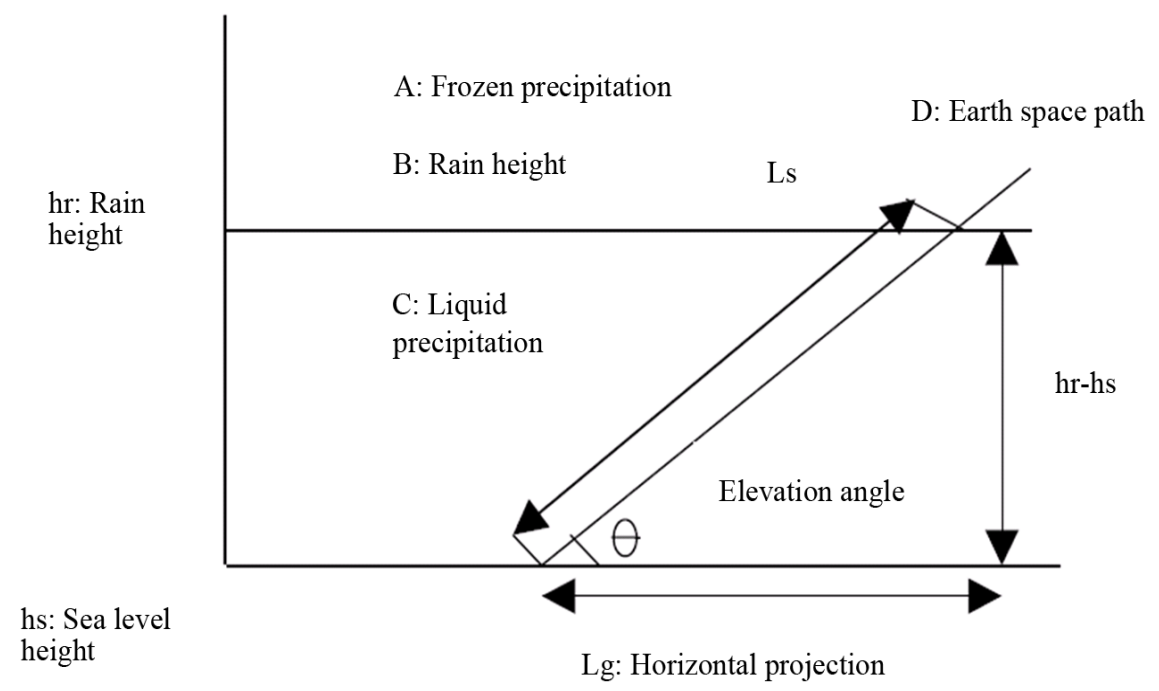

Figure 1. Schematic presentation of Earth-space path giving the parameters to be input into the attenuation prediction process [8]. 


$$
R_{0.01}=\frac{1}{1+0.78 \sqrt{\frac{L_{G} \gamma(R)}{f}-0.38\left(1-\mathrm{e}^{-2 L_{G}}\right)}}
$$

Step 7: Calculate the vertical adjustment factor, $v_{0.01}$, for $0.01 \%$ of the time:

$$
\begin{aligned}
& \zeta=\tan ^{-1}\left(\frac{h_{R}-h_{s}}{L_{G} r_{0.01}}\right) \text { degrees } \\
& \text { For } \xi>0, L_{R}=\frac{L_{G} r_{0.01}}{\cos \theta} \mathrm{km}
\end{aligned}
$$

Else,

$$
L_{R}=\frac{\left(h_{R}-h_{s}\right)}{\sin \theta} \mathrm{km}
$$

if $|\varphi|<36^{\circ}$,

$x=36-|\varphi|$ degrees

Else: $x=0$ degrees

$$
v_{0.01}=\frac{1}{1+\sqrt{\sin \theta}\left(31\left(1-\mathrm{e}^{-(\theta /(1+\chi))}\right) \frac{\sqrt{L_{R} \gamma_{R}}}{f^{2}}-0.45\right)}
$$

Step 8: The effective path length is:

$$
L_{E}=L_{R} v_{0.01}(\mathrm{Km})
$$

Step 9: The predicted attenuation exceeded for $0.01 \%$ of an average year is obtained from:

$$
A_{0.001}=g(R) L_{E}(\mathrm{~dB})
$$

Step 10: The estimated attenuation to be exceeded for other percentages of an average year, in the range $0.001 \%$ to $5 \%$, is determined from the attenuation to be exceeded for $0.01 \%$ for an average year:

If $P \geq 1 \%$ or $|\varphi| \geq 36^{\circ}$,

$\beta=0$.

If $P<1 \%$ and $|\varphi|<36^{\circ}$ and $\theta \geq 25^{\circ}$,

$\beta=-0.005(|\varphi|-36)$

Otherwise: $\beta=-0.005(|\varphi|-36)+1.8-4.25 \sin \theta$

$$
A_{p}=A_{0.01}\left(\frac{p}{0.01}\right)^{-\left(0.655+0.0331 n(p)-0.0451 n\left(A_{0.01}\right)-\beta(1-p) \sin \theta\right)} \mathrm{dB}
$$

This method provides an estimate of the long-term statistics of attenuation due to rain. When comparing measured statistics with the prediction, allowance should be given for the rather large year-to-year variability in rainfall rate statistics [12].

The Crane Global attenuation model was developed for use on either Earth-space or terrestrial paths. It is based entirely on geophysical observations of rain rate, rain structure, and the vertical variation of atmospheric temperature. Rain is characteristically inhomogeneous in the horizontal, and a statistical model is required to provide an estimate of the effect of the homogeneity on the estimation of attenuation. Crane accomplished this model by a piecewise representation of the path profile by exponential functions. An adequate model results when two exponential functions are used to span the 0 to $22.5 \mathrm{~km}$ distance range, one from 0 to $d(R) \mathrm{km}$, the other from d(R) to $22.5 \mathrm{~km}$. The resulting attenuation model for a given rain rate is given by [10]: 


$$
\begin{gathered}
A_{T}(R, d)=\gamma(R)\left(\frac{\mathrm{e}^{y \delta(R)}-1}{y}-\frac{\mathrm{e}^{z d}-\mathrm{e}^{y \delta(R)}}{z} \mathrm{e}^{\alpha B}\right) \\
\delta(R)<d<22.5 \\
A_{T}(R, d)=\gamma(R)\left(\frac{\mathrm{e}^{y \delta(R)}-1}{y}\right) 0<d<\delta(R)
\end{gathered}
$$

where

$A_{T}=$ horizontal path attenuation $(\mathrm{dB})$;

$R=$ rain rate $(\mathrm{mm} / \mathrm{hr})$;

$$
\gamma(R)=\text { specific attenuation }=k R^{\alpha}(\mathrm{dB} / \mathrm{km}) .
$$

The remaining coefficients are the empirical constants of the piecewise exponential model:

$$
\begin{aligned}
& B=\ln (b)=0.83-0.17 \ln (R) \\
& C=0.026-0.03 \ln (R) \\
& \delta(R)=3.8-0.6 \ln (R) \\
& u=\frac{B}{\delta(R)}+c \\
& y=\alpha u
\end{aligned}
$$

The lower is in frequency, the better the propagation characteristic is available, and for the higher the band, the more bandwidth that is available [7]. Therefore, the competition is keen for this spectrum due to its propagation characteristics.

The frequency used for satellite communications which are located in super high frequency (SHF) and extremely high frequency (EHF) bands of the electromagnetic frequency spectrum are listed in Table 1.

The quality of ISDN conferencing is limited by the bandwidth of the connection, varying from $64 \mathrm{kbit} / \mathrm{s}$ (ISDN-1) to 2 Mbps (ISDN-30). ISDN provides Guaranteed Quality of Service (GQoS), which means that if for example a $384 \mathrm{kbit} / \mathrm{s}$ link is dialed then unless there is a fault condition the full $384 \mathrm{kbit} / \mathrm{s}$ will be provided for the duration of the conference.

Packet-based network such as Local Area Networks (LANs), Wide Area Networks (WANs), Metropolitan Area Networks (MANs) and the Internet all uses the Internet Protocol (IP) to pass information between networks. Disadvantage of IP transmission is that all data traffic has to compete for the available bandwidth. These networks are currently unable to offer Guaranteed Quality of Service (GQoS). Also the bandwidth requirement is higher (about 20\%) on IP networks than on ISDN networks because of the packetizing overhead of IP. The advantage of making an IP based videoconferencing is that many people already have a connection to an existing IP infrastructure. So making an IP based videoconferencing system is the cheapest solution [13].

Point-to-Point is the most basic architecture configuration for a video conferencing network. This configuration does not require a Call Server and does not allow conferencing of more than two endpoints, but it does allow for direct video and audio calls between endpoints within a cluster. Multipoint conferencing makes conferencing possible by acting as a bridge that interconnects calls from three or more video endpoints. It can be a

Table 1. Satellite frequency spectrum.

\begin{tabular}{cc}
\hline Frequency band & Range (GHz) \\
\hline L-Band & $1-2$ \\
S-Band & $2-4$ \\
C-Band & $4-8$ \\
X-Band & $8-12$ \\
Ku-Band & $12-18$ \\
Ka-Band & $20-30$ \\
\hline
\end{tabular}


standalone hardware device, but some dedicated video conferencing have embedded multipoint conferencing functions. It also allows endpoints using different CODECs to participate in the same video conferencing session.

Gateways required in order for video conferencing endpoints to communicate with the Public Switched Telephone Network (PSTN) or a legacy network. A Gateway is a standalone hardware device that provides access to other larger IP networks and/or circuit switched telephone networks like the PSTN [14].

The signal is transmitted from one site via the uplink to WINDS and received to other site via the downlink. WINDS acts as a bent pipe, receiving and then re-transmitting to the second site. If the transmission suffers rain fades, both the uplink and the downlink are affected. In this project, the measured two-way loss rate is referred to as IP packet loss rate. For satellite transmissions, a video codec (a device or program that compresses data to enable faster transmission and decompresses received data) with high compression efficiency is required [15].

\section{Methods and Data Presentation}

The raindrop DSD was measured by an impact type disdrometer RD-80 manufactured by Disdromet ltd which is the advanced version of the RD-69 model developed by Joss and Waldogel installed at ICT master's program lab of University of Rwanda/Huye Campus $\left(2.6^{\circ} \mathrm{S}, 29.7^{\circ} \mathrm{E}\right)$ [23]. The measurements of DSD were taken with a time interval of 1 minute for better resolution of rain rates.

The Disdrometer RD-80 is a Joss and Waldvogel impact type instrument that measures raindrop size distribution continuously and automatically. RD-80 measures the drop size using a Styrofoam cone with a sampling cross-sectional area of $50 \mathrm{~cm}^{2}$ and has the ability to transform the vertical momentum of the drops to an electrical signal whose amplitude is a function of the drop diameter [16].

The University of Rwanda/ Huye Campus is located at $2.6^{\circ} \mathrm{S} 29.7^{\circ} \mathrm{E}$ in Huye district southern province of Rwanda. The following Figure 2 is the map indicating where these data have been collected on a daily basis.

The RD-80 Disdrometer consists of 2 units (see Figure 3). The processor and the sensor are exposed to rain. A cable, 20 meter long, is used to connect the sensor and the processor. The sensor transforms the momentum of an impacting drop into an electric pulse, whose amplitude is roughly proportional to the momentum. The sensor consists of a cylindrical metal housing, containing an electromechanical transducer and an amplifier module. The processor contains circuitry to eliminate unwanted signals, mainly due to acoustic noise to reduce the $90 \mathrm{~dB}$ dynamic range of the sensor signal and to digitize it into a 7-bit code at the output for every drop, hitting the sensitive surface of the sensor [18]. One of its drawbacks is that it underestimates the number of small drops in heavy rain due to the disdrometer "dead time". The detection of smaller drops is also suppressed in the presence of background noise. Further, drops larger than 5.0 to $5.5 \mathrm{~mm}$ diameter cannot be distinguished by the disdrometer [19].

The sensor consists of an electromechanical unit and an amplifier module in common housing. A conical

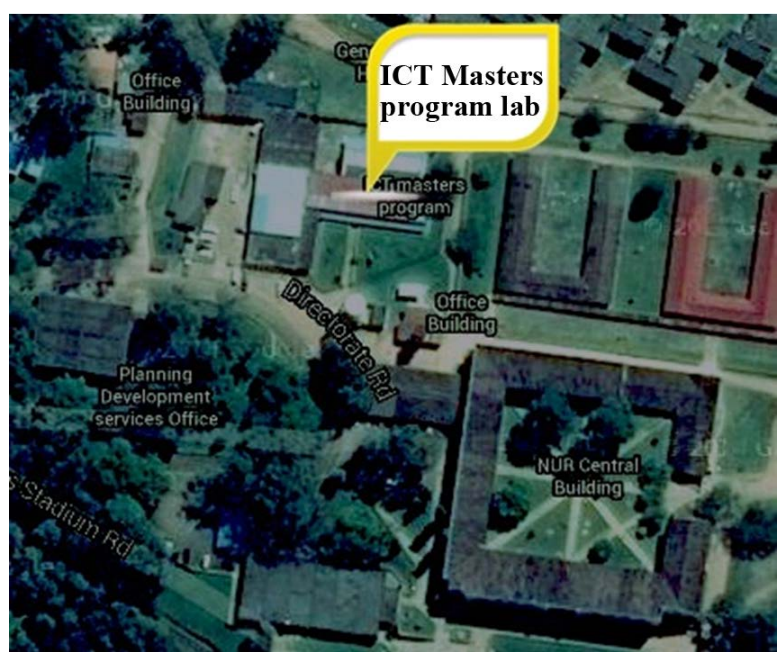

Figure 2. ICT master's program lab at UR-Huye campus [17]. 


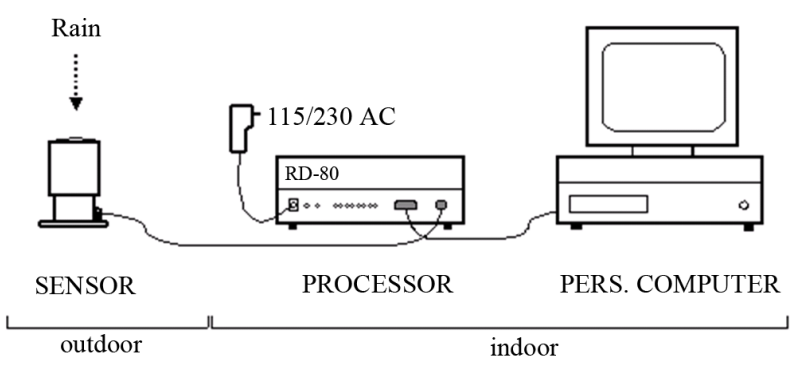

Figure 3. Disdrometer for Raindrops Type RD-80 [21].

Styrofoam body is used to transmit the mechanical impulse of an impacting drop to a set of moving coils in a magnetic field. The Styrofoam body and the two coils are fixed together rigidly. At the impact of a drop the Styrofoam body together with the two coils moves downwards and a voltage is induced in the sensing coil. This voltage is amplified and applied to the driving coil such that a force counteracting the movement is produced. As a consequence the excursion is very small and it takes very little time for the system to return to its original resting position and therefore to get ready for the next impact of another drop. The amplitude of the pulse at the output of the amplifier is the measure of the size of the drop that caused it [20].

$\mathrm{RD}-80$ is considered to be a reference instrument in measuring the DSD at the ground. Basically the RD-80 transforms the energy of falling drops into electric current. The RD-80 derives the size of the individual drops from the measured impact velocity of the drops through an empirical nonlinear relationship between fall velocity and drop diameter in still air [16]. RD-80 distinguishes 127 classes of drop diameter which are then sorted into 20 size intervals ranging from $0.3 \mathrm{~mm}$ to $5 \mathrm{~mm}$ using DATA LOGGING program to reduce amount of data and get statistically meaningful samples [22]. It should be noted that the accuracy of Joss-Waldvogel's disdrometer is sensitive to background noise, for this reason it could underestimate the small raindrops in heavy rain when the Styrofoam cone is hit by many more drops [16].

The sensor is mostly placed on the roof of building and away from equipment that can generate electromagnetic fields to minimize errors due to background noise and interferences [16] [22].

Drop size distribution can be computed by first calculating the quantity $N\left(D_{i}\right)$ the number of density of drops of the diameter corresponding to size class $i$ per unit volume from the data for every drop size according to following the formula:

$$
N\left(D_{i}\right)=\frac{n_{i}}{F \times t \times v\left(D_{i}\right) \times \Delta D_{i}}
$$

where $n_{i}$ is the number of drops measured in drop size;

$D_{i}$ is the average diameter of the drops in class $i$;

$F$ is the size of sensitive surface of the disdrometer;

$v\left(D_{i}\right)$, the fall velocity of the drop with the diameter $D_{i}$;

and $\Delta D_{i}$ the diameter interval of drop class $i$.

The size of the sensitive area of the disdrometer sensor is given by the manufacturer as $50 \mathrm{~cm}^{2}$. Rain rate as an integral rain parameter is calculated as follow [23]:

$$
R=\frac{\pi}{6} \times \frac{3.6}{10^{3}} \times \frac{1}{F \times t} \times \sum_{i=1}^{20}\left(n_{i} \times D_{i}^{3}\right)
$$

And the rainfall

$$
R A=\frac{(R \times t)}{3600}
$$

The accumulated rainfall is $R T=\sum R A$

\section{Data Analysis and Interpretation}

The output data from the Disdrometer is being logged automatically into a computer as per the temporal resolu- 
tion of the data is set for. The data cables from the Disdrometer have been connected to one computer and the data has been logged every minute to this computer. This instrument measures and record the rain drop size distributions falling on the sensitive surface of the sensor for each time interval and are easily analyzed using Microsoft Excel. Thus, using Drop Size Distribution, we can simply calculate different rain parameters such as rain intensity, rain amount and total rain amount since the start of measurement and the cumulative distribution function of one minute rain rate for July and August 2014.

Data was recorded during two months period of July and August 2014, which are not heavy rainy months in Rwanda especially here in UR-Huye Campus where data was collected. Rain rate exceedance characteristics need to be analyzed in order to provide information regarding the rain rate of $0.01 \%$ time of the year to attain $0.01 \%$ link reliability of video conference system. The daily rain rate curves have been plotted in the Figure 4 below.

The rainfall rates exceeded for $0.01 \%$ of the time found at UR-HUYE Campus are $1 \mathrm{~mm} / \mathrm{hr}$ of July and 13.5 $\mathrm{mm} / \mathrm{hr}$ of August 2014. The results show that for low rainfall rate, we have high percentages of time and viceversa.

The cumulative distribution of rain rates exceeded for percentages of the time in July and August 2014 for UR-Huye Campus is given in the Figure 5 below.

Specific attenuation $\gamma(\mathrm{dB} / \mathrm{km})$ is obtained from the rain rate $R(\mathrm{~mm} / \mathrm{h})$ using Equation 2.4 where $k$ and $\alpha$ are frequency-dependent power law parameters and $R_{0.01}$ is the rain rate exceeded for $0.01 \%$ of the time.

The frequency-dependent coefficients $k$ and $\alpha$ are given in the table below for linear polarizations (horizontal: $\mathrm{H}$, vertical: V) and horizontal paths [24].

For linear and circular polarization, and for all path geometries, $k$ and $\alpha$ can be calculated from the values in Table 2 using the following equations [24]:

$$
\begin{gathered}
k=\left[k_{H}+k_{V}+\left(k_{H}-k_{V}\right) \cos ^{2} \theta \cos 2 \tau\right] / 2 \\
\alpha=\left[k_{H} \alpha_{H}+k_{V} \alpha_{V}+\left(k_{H} \alpha_{H}-k_{V} \alpha_{V}\right) \cos ^{2} \theta \cos 2 \tau\right] / 2 k
\end{gathered}
$$

where $\theta$ is the path elevation angle and $\tau$ is the polarization tilt angle relative to the horizontal ( $\tau=45^{\circ}$ for circular polarization). For circular polarization, replacing in the equations above by $\tau=45^{\circ}$, we obtain:

$$
k=\left[k_{H}+k_{V}\right] / 2
$$

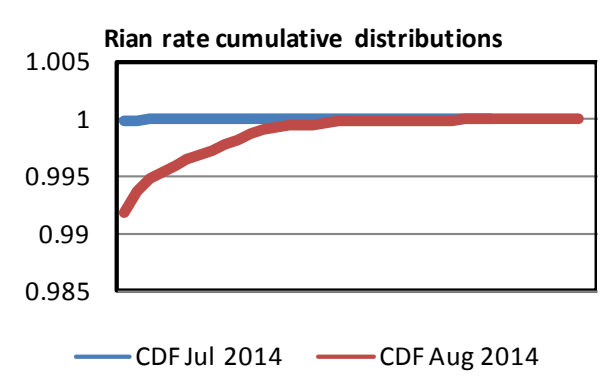

Figure 4. Daily rain rate exceedance curves of UR-HUYE campus region.

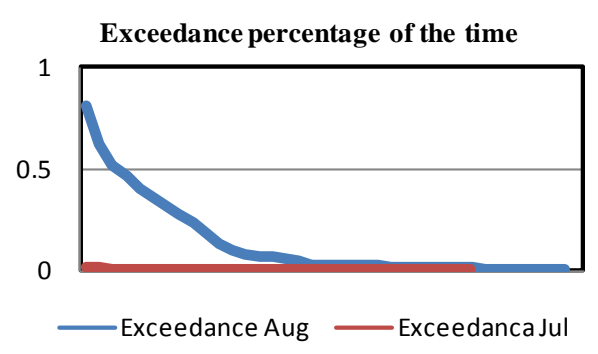

Figure 5. Exceedance percentage of the time UR-Huye Campus region. 
Table 2. Frequency-dependent coefficients for estimating specific rain attenuation.

\begin{tabular}{ccccc}
\hline $\begin{array}{c}\text { Frequency } \\
(\mathrm{GHz})\end{array}$ & $k_{H}$ & $k_{V}$ & $\alpha_{H}$ & $\alpha_{V}$ \\
\hline 12 & 0.01882 & 0.01680 & 1.2168 & 1.1994 \\
14 & 0.0309 & 0.028 & 1.1755 & 1.1515 \\
20 & 0.07504 & 0.06898 & 1.0995 & 1.0663 \\
30 & 0.1864 & 0.1673 & 1.0202 & 0.9974 \\
\hline
\end{tabular}

Table 3. Calculation of power law parameters and specific attenuation of the month of July and August 2014.

\begin{tabular}{ccccc}
\hline & \multicolumn{2}{c}{ July 2014 } & \multicolumn{2}{c}{ August 2014 } \\
\hline Frequency (GHz) & 20 (downlink) & 30 (uplink) & 20 (downlink) & 30 (uplink) \\
\hline$k_{H}$ & 0.07504 & 0.1864 & 0.0750 & 0.1864 \\
$k_{V}$ & 0.06898 & 0.1673 & 0.06898 & 0.1673 \\
$\alpha_{H}$ & 1.0995 & 1.0202 & 1.0995 & 1.0202 \\
$\alpha_{V}$ & 1.0663 & 0.9974 & 1.0663 & 0.9974 \\
$R_{0.01} \mathrm{~mm} / \mathrm{hr}$ & 1 & 1 & 13.5 & 13.5 \\
$\gamma \mathrm{dB} / \mathrm{km}$ & 0.072 & 0.177 & 1.21 & 2.44 \\
\hline
\end{tabular}

$$
\alpha=\left[k_{H} \alpha_{H}+k_{V} \alpha_{V}\right] / 2 k
$$

with $\alpha$ calculated from $k$ as shown above.

Thus, the previous equations help us solving the Equation 2.4. The main objective of this paper was to find the specific attenuation which has been calculated based on frequency band allocated to video conferencing (30 GHz uplink and $20 \mathrm{GHz}$ downlink) at UR-Huye campus during July and August 2014. Working on both ka and ku-band in order to highlight the effect of high frequencies apart from rain on signal fade; especially in video conference systems as our major concern, Table 3 shows the results.

From the table above, we can see that the rain rate exceeding $0.01 \%$ for the data collected is $1 \mathrm{~mm} / \mathrm{hr}$ for July and $13.5 \mathrm{~mm} / \mathrm{hr}$ for August whereas it is $42 \mathrm{~mm} / \mathrm{h}$ for the ITU-R model in region K where Rwanda is located. However the data considered was collected for only 2 months and it is not accurate to propose a suitable rain attenuation model for the region under study due the facts related to insufficient procured data (only July 2014 and August 2014) and a very small region of study. Consequently, it can be better before concluding that the model doesn't fit well, to have other data for all over the year and this can be done for at least three years.

\section{Conclusions}

The results by using the ITU-R attenuation method approach for prediction of rain attenuation on microwave links used in video conferencing technology have been evaluated using two months rain data. Attenuation was evaluated with respect to rainfall rate.

In this paper, the results of rain attenuation measurement during two months of July and August are presented and compared with ITU-R model results. According to the calculated rain rate, the probability of rainfall rate exceeding $1 \mathrm{~mm} / \mathrm{hr}$ of July and $13.5 \mathrm{~mm} / \mathrm{hr}$ of August is approximately $0.01 \%$ of each month respectively, so 1 $\mathrm{mm} / \mathrm{hr}$ and $13.5 \mathrm{~mm} / \mathrm{hr}$ effectively do not represent heavy rain, hence it's observed that the southern region where UR-Huye campus resides receives the least rainfall in the months of July and August. Rain rates statistics determined using rain intensity recorded during two months of July and August shows that the obtained rain rates were different from those proposed by ITU-R. According to its rain zoning, the rainfall rate $\mathrm{R}_{0.01}$, which exceeds for $0.01 \%$ of time predicted K-region where Rwanda belongs to, is $42 \mathrm{~mm} / \mathrm{hr}$, which indicates that ITU$\mathrm{R}$ predicted and estimated values are not quite good enough to predict the attenuation in microwave signals in this region. 
Actually during the months where data was collected, there is no enough rain to have effects on videoconferencing system. In the future, to continue verifying the ITU-R model prediction in the region where the Rwanda region is located, we want to analyze data for the season comprising the months of September, October, November and December. From there it can be possible to compare our data and the ITU-R model.

\section{References}

[1] http://www.microlink.co.in/solutions/audio-visual-solution/video-conferencing-solution

[2] http://www.wisegeek.org/what-is-video-conferencing.htm

[3] Castanet, L. (2002) Simulation of the Performance of a Ka-Band V-SAT Videoconferencing System with Uplink Power Control and Data Rate Reduction to Mitigate Atmospheric Propagation Effects. J. Sat. Comm.

[4] Ma, T. (2014) QoS Provisioning by Power Control for Video Communication via Satellite Links. Nanyang Technological University, Singapore.

[5] Sofa, S.M.B. (2013) Troposphere Amplitude Scintillation for Earth to Satellite Link in Tropical Climate. Malaysia University of Technology, Malaysia.

[6] http://en.wikipedia.org/wiki/Terminal_velocity

[7] Mohamad Sobiron, M.F.B. (2009) Rain Rate Attenuation Prediction Model at 12 GHz in Melaka. Melaka Technical University of Malaysia, Melaka.

[8] Harun, H., Agil, H. and Rashid, R.A. (2013) Improvements of Signal Gain for Measat-2 and Measat-3 Using Orbital Diversity under Rain Attenuation: A Simulation Approach. International Journal of Scientific and Engineering Research, 4, 367-372.

[9] Ojo, J.S., Ajewole, M.O. and Sarkar, S.K. (2008) Rain Rate and Rain Attenuation Prediction for Satellite Communication in Ku and Ka Bands over Nigeria. Progress in Electromagnetics Research B, 5, 207-223. http://dx.doi.org/10.2528/PIERB08021201

[10] Das, S., Maitra, A. and Shukla, A.K. (2010) Rain Attenuation Modeling in the 10-100 GHz Frequency Using Drop Size Distributions for Different Climatic Zones in Tropical India. Progress in Electromagnetics Research B, 25, 211224.

[11] (2005) ITU-R Recommendation p.838-2. Specific Attenuation Model for Rain for Use in Prediction Methods. International Telecommunication Union, Geneva.

[12] Singh, M.S.J. (2007) Rainfall Attenuation and Rainfall Rate Measurements in Malaysia, Comparison with Prediction Models. School of Electrical and Electronics Engineering, University of Sciences, Penang.

[13] https://www.nsa.gov/ia/_files/factsheets/i73-021r-2009

[14] Roy, S.S., Datta, R.K. Bhatia, R.C. and Sharma, A.K. (2005) Drop Size Distributions of Tropical Rain over South India. Geofizika, 22, 105-131.

[15] Ma, T., Lee, Y.H., Winkler, S. and Ma, M. (2015) QoS Provisioning by Power Control forVideo Communication via Satellite Links. International Journal of Satellite Communications and Networking, 33, 259-275. http://dx.doi.org/10.1002/sat.1087

[16] Liu, X.C., Gao, T.C. and Liu, L. (2013) A Comparison of Rainfall Measurements from Multiple Instruments. University of Science and Technology, Nanjing.

[17] https://www.google.rw/maps/place/University+of+Rwanda/@-2.6154801,29.7465159,2214m/data=!3m1!1e3!4m2!3m 1!1s0x0:0x5e3baaf0d5aded1b!6m1!1e1

[18] (1992-1999-2003) Recommendation ITU-R p.838-2. Specific Attenuation Model for Rain for Use in Prediction Methods. International Telecommunication Union, Geneva.

[19] https://www.itu.int/dms pubrec/itu-r/rec/p/R-REC-P.838-2-200304-S!!PDF-E.pdf

[20] Harikumar, R. (2003) Experimental Techniques, Observation Stations and Data Availability, p. 52.

[21] http://distromet.com/content/56/55/product-description-general

[22] Disdrometer Ltd., Switzerland (2002) Disdrometer RD-80 Instruction Manual.

[23] Crane, R.K. (1977) Prediction of the Effects of Rain on Satellite Communication System. Proceedings of the IEEE, 65, 456-474. http://dx.doi.org/10.1109/PROC.1977.10498

[24] Disdrometer Ltd. (2010) Disdrometer RD-80 User Guide for Disdrodata 2.1. Data Acquisition on Personal Computer for Disdrometer RD-80 for Microsoft WINDOWS 2000, XP, Vista 7. 